\title{
ON THE AXIALLY SYMMETRIC FLOW AROUND A NEW FAMILY OF HALF-BODIES*
}

\author{
BY \\ A. VAN TUYL \\ Naval Ordnance Laboratory, Silver Springs, Md.
}

1. Introduction and discussion. A recent paper by A. Weinstein [1] $\dagger$ includes a discussion of the flow of a perfect incompressible fluid corresponding to a uniform discsource at right angles to a uniform parallel flow from infinity. There is a stream surface in the form of a half-body under certain conditions whose shape depends on a single parameter. As the radius of the disc goes to zero, with both the total source strength and the velocity at infinity held constant, the half-body continually decreases in bluntness until the form of the well-known Blasius-Fuhrmann half-body (cf. [1], for example) is reached. When the radius increases under the same conditions, an upper value is reached at which the nose coincides with the disc.

The object of the present paper is to make a combined numerical and analytical study of these half-bodies, and some features of the corresponding velocity distributions. A set of graphs is obtained by means of which the nose of any given profile can be plotted; representative profiles are shown. Curves are also given which show the position and the magnitude of the maximum velocity on the surface as functions of the halfbody. It follows from Bernoulli's theorem that the pressure is least at the point of maximum velocity, hence the danger of cavitation is greatest there in an actual flow. All calculations are made using expressions for the stream function and velocity components in terms of elliptic integrals.

The total source strength will be denoted by $m$, the radius of the disc by $b$, and the velocity at infinity by $U$. Cylindrical coordinates $x, \rho$ will be used with $x$ measured in the direction of the parallel flow and with the center of the disc at $x=0, \rho=0$. Then as given in [1], the stream function for the total flow is

$$
\psi=\frac{1}{2} U \rho^{2} \mp 2 m \rho b^{-1} \int_{0}^{\infty} e^{-|x| t} J_{1}(\rho t) J_{1}(b t) t^{-1} d t+ \begin{cases}0, & x \leq 0 \\ -2 m, & x \geq 0\end{cases}
$$

where $J_{1}(z)$ is the Bessel function of the first kind of order one. The additive constant is chosen so that the stream function vanishes on the line $\rho=0, x<0$. It is shown in [1] that $b \leq(2 m / U)^{1 / 2}$ is the necessary and sufficient condition for $\psi=0$ to be the equation of a half-body. The $b$-independent quantity $(2 m / U)^{1 / 2}$ is the intercept of the profile on the $\rho$-axis, and will be denoted by $\rho_{0}$. The half-body is asymptotic to the cylinder $\rho=2^{1 / 2} \rho_{0}$.

The components of velocity in the $x$ - and $\rho$-directions, respectively, are

$$
u=\rho^{-1} \frac{\partial \psi}{\partial \rho}, \quad v=-\rho^{-1} \frac{\partial \psi}{\partial x} .
$$

*Received March 29, 1949.

$\nmid$ Numbers in brackets refer to the references at the end of the paper. 
Concerning the stream function for the uniform disc-source, some errors have occurred in the literature. The stream function was given incorrectly by Beltrami [2], who apparently overlooked the many-valued character of the stream function for the ring and integrated a branch which is continuous across the interior of the ring. This error was reproduced by Webster [3, p. 367]; Lamb [4, p. 239] has given it incompletely, but correctly as far as it is given. The flow corresponding to a ring of sources has been investigated by several others. However, it seems that the fundamental fact that the corresponding stream function is many-valued has not been mentioned except in [1].

We now make the substitution $b t=s$, and introduce the notation

$\psi^{*}=m^{-1} \psi, \quad \lambda=b^{-1} \rho_{0}, \quad \xi=b^{-1} x, \quad \eta=b^{-1} \rho, \quad u^{*}=U^{-1} u, \quad v^{*}=U^{-1} v$.

For (1.1) and (1.2), respectively, we obtain

$$
\begin{gathered}
\psi^{*}=\frac{\eta^{2}}{\lambda^{2}} \mp 2 \eta \int_{0}^{\infty} e^{-|\xi| s} J_{1}(\eta s) J_{1}(s) s^{-1} d s+\left\{\begin{array}{cc}
0, & \xi \leq 0 \\
-2, & \xi \geq 0
\end{array}\right. \\
u^{*}=\frac{\lambda^{2}}{2 \eta} \frac{\partial \psi^{*}}{\partial \eta}, \quad v^{*}=-\frac{\lambda^{2}}{2 \eta} \frac{\partial \psi^{*}}{\partial \xi} .
\end{gathered}
$$

From (1.4) and (1.5), it follows that

$$
\begin{gathered}
u^{*}=1 \mp \lambda^{2} \int_{0}^{\infty} e^{-|\xi| s} J_{0}(\eta s) J_{1}(s) d s, \quad\left\{\begin{array}{l}
\xi \leq 0 \\
\xi \geq 0
\end{array}\right. \\
v^{*}=\lambda^{2} \int_{0}^{\infty} e^{-|\xi| s} J_{1}(\eta s) J_{1}(s) d s .
\end{gathered}
$$

Given $\lambda$, the equation of the profile $\psi=0$ in the $\xi \eta$-plane is

$$
\lambda^{-2}= \pm 2 \eta^{-1} \int_{0}^{\infty} e^{-|\xi| s} J_{1}(\eta s) J_{1}(s) s^{-1} d s+\left\{\begin{array}{ll}
0, & \xi \leq 0 \\
2 \eta^{-2}, & \xi \geq 0
\end{array}=T(\xi, \eta) .\right.
$$

We introduce also

$$
u_{0}^{*}=1+\left(\lambda^{2} T\right)^{-1}\left(u^{*}-1\right), \quad v_{0}^{*}=\left(\lambda^{2} T\right)^{-1} v^{*}, \quad w_{0}^{*}=\left(u_{0}^{* 2}+v_{0}^{* 2}\right)^{1 / 2}
$$

By $1.8, w_{0}^{*}$ at a point $(\xi, \eta)$ is the velocity magnitude along the profile which passes through it.

It is possible to express (1.6), (1.7), and (1.8) in terms of elliptic integrals, and this is done here. It was noted by Weber [5] that integrals of the form of (1.8) can be evaluated in terms of elliptic integrals, although the reduction was not carried out. Both complete and incomplete elliptic integrals of the first and second kinds are present in (1.6) and (1.8), while the evaluation of (1.7) involves only complete elliptic integrals. As carried out here, (1.7) and (1.8) are evaluated separately, and (1.6) is expressed as a linear combination of (1.7) and (1.8) and a third integral which can be evaluated in terms of complete elliptic integrals. We thus arrive at expressions for (1.6) and (1.7) of the form $a_{1} K+b_{1} E+c T+d$ and $a_{2} K+b_{2} E$, where $K$ and $E$ are the complete elliptic integrals of the first and second kinds, and the coefficients are simple algebraic 
functions of $\xi$ and $\eta$. It turns out that (1.8) is most simply expressed in terms of $K$ and $(K-E) / k^{2}=D$, where $k$ is the modulus, and (1.6) and (1.7) in terms of $K$ and $(2 D-K) / k^{2}=C$. Both $C$ and $D$ are tabulated in Jahnke and Emde, [6]. The tables of $K$ and $E$ are the most accurate, however, and are used here throughout.

In the first part of the computations, $T(\xi, \eta)$ is calculated as a function of $\xi$ for several fixed values of $\eta$, and the results are plotted. Then making the substitution $\xi T^{1 / 2}=x$, $\eta T^{1 / 2}=\cdot \rho$, a second set of graphs is made from the first showing $T$ as a function of $x$ for several fixed values of $\rho$ between 0 and $2^{1 / 2}$. It is evident that the profiles $T(x, \rho)=$ constant all have the $\rho$-intercept unity, and are asymptotic to $\rho=2^{1 / 2}$. In addition, $(\partial T / \partial x)_{\rho}$ has been found as a function of $\rho$ at $T=0$, for $0 \leq \rho \leq 2^{1 / 2}$, and at $T=1$, $x=0$, where $0 \leq \rho \leq 1$. The first is a simple algebraic expression, the second involves elliptic integrals. Figure 1 shows $T$ as a function of $x$ for several values of $\rho$, while Fig. 2 gives some of the profiles $T=$ constant.

To investigate the position of maximum velocity along the profile, the function $R(\xi, \eta)=2^{-1} u_{0}^{*}\left(\partial \mathrm{w}_{0}^{* 2} / \partial \xi\right)_{T^{\prime}}$ has been obtained in terms of elliptic integrals. It is expressed in the form $a u_{0}^{*^{2}}+b u_{0}^{*} v_{0}^{*}+c v_{0}^{*^{2}}$, where the coefficients are linear combinations of complete elliptic integrals and $T(\xi, \eta)$. Evidently, the curve $R(\xi, \eta)=0$ intersects a profile $T=$ constant at the point of maximum velocity. To find points of $R(\xi, \eta)=0$, $u_{0}^{*}$ and $v_{0}^{*}$ have first been calculated as functions of $\xi$ for the same values of $\eta$ taken before; then the zero of $R(\xi, \eta)$ has been found for each $\eta$. The curve begins at $\xi=0$, $\eta=1$ and crosses the axis at some $\eta>1$. This value of $\eta$ has been found numerically. From the graphs of $T(\xi, \eta)$ and the transformation $x=\xi T^{1 / 2}$, the value of $x$ at which maximum velocity occurs, denoted by $x_{m}$, is found as a function of $T$. It is found that $x_{m}(T)$ is positive for $0 \leq T<0.819$, and negative for $0.819<T<1$. At $T=0$, which defines the Blasius-Fuhrmann half-body, $x_{m}(T)$ is equal to $6^{-1 / 2}$. Further details are that $d x_{m} / d T=-(13 / 48) 6^{1 / 2}$ at $T=0$, and $d x_{m} / d T=+0$ at $T=1-0$. When measured from the nose instead of from the cross section of radius unity, $x_{m}$ seems to be always decreasing. The plot of $x_{m}$ as a function of $T$ is shown in Fig. 3. The portion of the curve between $T=0.819$ and $T=1$ is below the axis, but too close to be distinguished from it.

Finally, $w_{0 \max }^{*}$ is plotted as a function of $T$ in Fig. 4 . It becomes infinite at $T=1$ and is equal to $2 / 3^{1 / 2}$ at $T=0$. $^{*}$

2. Evaluation of $T(\xi, \eta)$. Setting $\mu=0, \nu=1$ in Watson [7, p. 389, formula (1)], we obtain

$$
\int_{0}^{\infty} e^{-\xi t} J_{1}(\eta t) J_{1}(t) t^{-1} d t=\eta\left(2 \pi \xi^{2}\right)^{-1} \int_{0}^{\pi} F\left(1,3 / 2 ; 2 ;-\mathrm{p}^{2} \xi^{-2}\right) \sin ^{2} \varphi d \varphi,
$$

where $F(a, b ; c ; z)$ is the hypergeometric function, and $\mathrm{p}^{2}=1+\eta^{2}-2 \eta \cos \varphi$. We have

$$
\begin{aligned}
F(1,3 / 2 ; 2 ; z) & =2 \sum_{n=1}^{\infty} \frac{1 \cdot 3 \cdots(2 n-1)}{2 \cdot 4 \cdots(2 n)} z^{n-1}, \\
& =2 z^{-1}\left[(1-z)^{-1 / 2}-1\right] .
\end{aligned}
$$

${ }^{*}$ The author is indebted to Miss C. Brudno and Miss G. Fulton for the drawings and numerical calculations. 
Thus,

$$
\begin{aligned}
\int_{0}^{\infty} e^{-\xi t} J_{1}(\eta t) J_{1}(t) t^{-1} d t & =\frac{\eta}{\pi} \int_{0}^{\pi} \frac{\sin ^{2} \varphi d \varphi}{1+\eta^{2}-2 \eta \cos \varphi} \\
& -\frac{\xi \eta}{\pi} \int_{0}^{\pi} \frac{\sin ^{2} \varphi d \varphi}{\left(1+\eta^{2}-2 \eta \cos \varphi\right)\left(\xi^{2}+1+\eta^{2}-2 \eta \cos \varphi\right)^{1 / 2}} \cdot
\end{aligned}
$$

We find that

$$
\begin{aligned}
\int_{0}^{\pi}\left(1+\eta^{2}-2 \eta \cos \varphi\right)^{-1} \sin ^{2} \varphi d \varphi & =\frac{\pi}{2 \eta^{2}}, & & \eta \geq 1, \\
& =\frac{\pi}{2}, & & \eta \leq 1 .
\end{aligned}
$$

We substitute $\cos \varphi=2 t^{2}-1$ into the second integral on the right and set $4 \eta\left[\xi^{2}+\right.$ $\left.(\eta+1)^{2}\right]^{-1}=k^{2}, 4 \eta(1+\eta)^{-2}=l^{2}$. Clearly $k^{2} \leq l^{2} \leq 1$. Then for all $\eta>0$,

$$
\begin{aligned}
& \int_{0}^{\pi}\left(1+\eta^{2}-2 \eta \cos \varphi\right)^{-1}\left(\xi^{2}+1+\eta^{2}-2 \eta \cos \varphi\right)^{-1 / 2} \sin ^{2} \varphi d \varphi \\
& =8(1+\eta)^{-2}\left[\xi^{2}+(1+\eta)^{2}\right]^{-1 / 2}\left[l^{-2} \int_{0}^{1} t^{2}\left(1-t^{2}\right)^{-1 / 2}\left(1-k^{2} t^{2}\right)^{-1 / 2} d t\right. \\
& \left.\quad-\left(l^{-2}-1\right) \int_{0}^{1} t^{2}\left(1-l^{2} t^{2}\right)^{-1}\left(1-t^{2}\right)^{-1 / 2}\left(1-k^{2} t^{2}\right)^{-1 / 2} d t\right]
\end{aligned}
$$

Finally, to reduce the integrals on the right to standard elliptic integrals, we substitute $t=\mathrm{sn}(u, k)$. We have*

$$
\int_{0}^{1} t^{2}\left(1-t^{2}\right)^{-1 / 2}\left(1-k^{2} t^{2}\right)^{-1 / 2} d t=\int_{0}^{K} \mathrm{sn}^{2}(u, k) d u=(K-E) / k^{2},
$$

where $K$ and $E$ are the complete elliptic integrals of the first and second kinds, respectively. Similarly,

$$
\int_{0}^{1} t^{2}\left(1-l^{2} t^{2}\right)^{-1}\left(1-t^{2}\right)^{-1 / 2}\left(1-k^{2} t^{2}\right)^{-1 / 2} d t=\int_{0}^{K}\left(1-l^{2} \operatorname{sn}^{2} u\right)^{-1} \operatorname{sn}^{2} u d u .
$$

With sn $(\delta, k)=l / k$,

$\int_{0}^{K}\left(1-l^{2} \operatorname{sn}^{2} u\right)^{-1} \operatorname{sn}^{2} u d u=\left(k^{2} \operatorname{sn} \delta \operatorname{cn} \delta \operatorname{dn} \delta\right)^{-1} \int_{0}^{K} \frac{k^{2} \operatorname{sn} \delta \operatorname{cn} \delta \operatorname{dn} \delta \operatorname{sn}^{2} u d u}{1-k^{2} \operatorname{sn}^{2} \delta \operatorname{sn}^{2} u}$.

The integral on the right of $(2.8)$ is $\Pi(K, \delta)$, the complete elliptic integral of the third kind with parameter $\delta$, having as its path of integration the segment of the real axis from 0 to $K$. We note that $\Pi(K, \delta)$ is doubly periodic in $\delta$ with the periods $2 K$ and $2 K^{\prime} i$. By [8, p. 523] we have

$$
\Pi(K, \delta)=n \pi i+K Z(\delta, k)
$$

*All formulas concerning elliptic functions and elliptic integrals used here are given in Whittaker and Watson [8, Ch. XXII]. 
where $Z(\delta, k)$ is the Jacobi Zeta function, and $n$ is an integer which depends on the determination of $\delta$.

We now make the substitution $\delta=K+i M$, and use the addition theorem, obtaining Jacobi's imaginary transformation

$$
\operatorname{sn} \delta=\frac{\mathrm{cn}(i M, k)}{\operatorname{dn}(i M, k)}=\frac{1}{\operatorname{dn}\left(M, k^{\prime}\right)}=l / k,
$$

where $k^{\prime 2}=1-k^{2}$. Since $k \leq k / l \leq 1$, it follows that $M$ is a quantity between $-K^{\prime}$ and $K^{\prime}$, plus multiples of $2 K^{\prime}$. Noting that $\Pi(K, \delta)$ and $Z(\delta, k)$ both tend to zero as

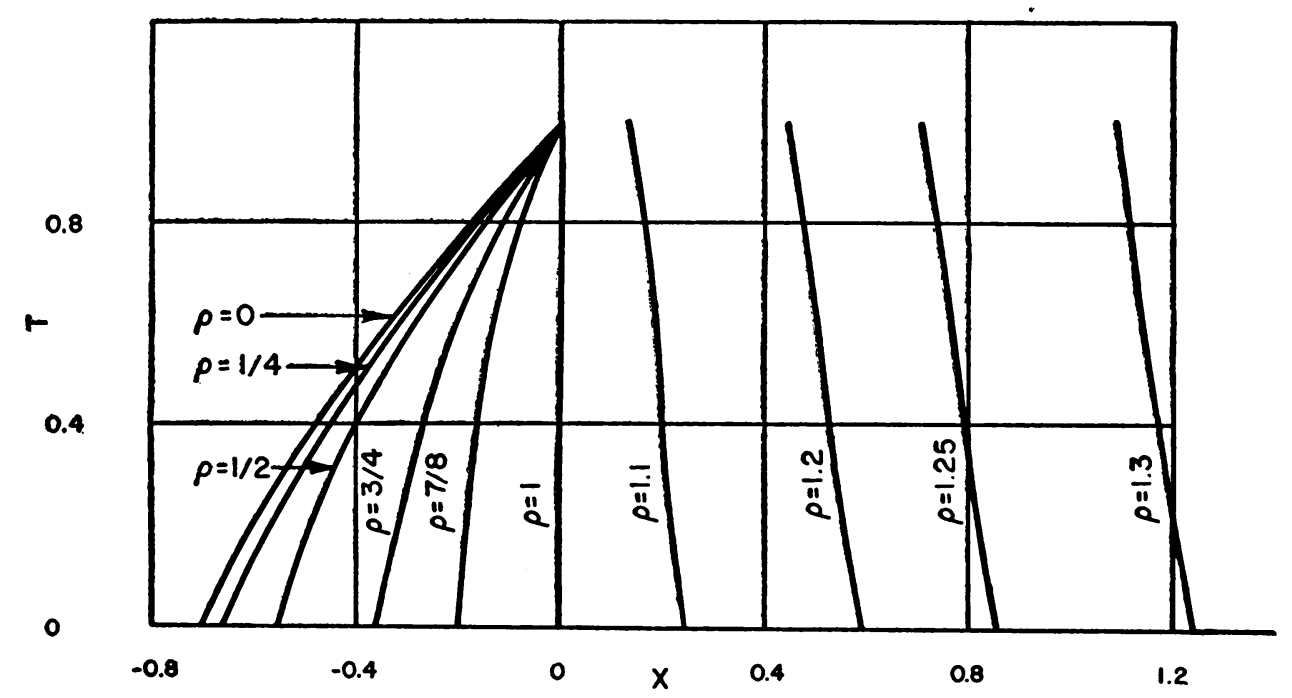

FIG. 1.

$M$ tends to zero, we find that $n=0$ for $-K^{\prime}<M<K^{\prime}$. We see that $\Pi(K, \delta)$ has discontinuities at $M=(2 m+1) K^{\prime}$, and that $\Pi(K, \delta)=0$ at $M=(2 m+1) K^{\prime}$. The discontinuous behavior of $\Pi(K, \delta)$ can also be seen from (2.8), since the left side becomes infinite at both $M=K^{\prime}-0$ and $M=K^{\prime}+0$, while cn $\delta$ changes sign at $M=K^{\prime}$.

All values of $M$ differing by multiples of $2 K^{\prime}$ are given by

$$
M=\int_{0}^{\varphi}\left(1-k^{\prime 2} \sin ^{2} \varphi\right)^{-1 / 2} d \varphi
$$

when $\varphi=\operatorname{arc} \sin \operatorname{sn}\left(M, k^{\prime}\right)=\arctan \left[\xi(\eta-1)^{-1}\right]$ runs through all values differing by multiples of $\pi$. Choosing $\varphi$ so that $\varphi=0$ for $\xi=0, \eta>1$, we see that $-K^{\prime}<M<$ $K^{\prime}$ when $\eta>1$. As a function of $\varphi, M$ is Legendre's incomplete elliptic integral of the first kind with modulus $k^{\prime}$. In the notation of Legendre for incomplete elliptic integrals, used in Jahnke and Emde,

$$
M=F\left(k^{\prime}, \varphi\right) .
$$

By $[8$, p. 518],

$$
Z(\delta, k)=E(\delta, k)-(E / K) \delta
$$


where $E(\delta, k)$ is the incomplete elliptic integral of the second kind with argument $\delta$. Using the addition theorem and Jacobi's imaginary transformation for $E(\delta, k)$, we obtain, for $-K^{\prime}<M<K^{\prime}$,

$$
\Pi(k, \delta)=i\left[k^{2} \frac{\mathrm{sn}\left(M, k^{\prime}\right) \mathrm{cn}\left(M, k^{\prime}\right)}{\operatorname{dn}\left(M, k^{\prime}\right)}+(K-E) M-K E\left(M, k^{\prime}\right)\right] .
$$

The notation is that of Whittaker and Watson, who write

$$
E\left(M, k^{\prime}\right)=\int_{0}^{M} \operatorname{dn}^{2}\left(u, k^{\prime}\right) d u .
$$

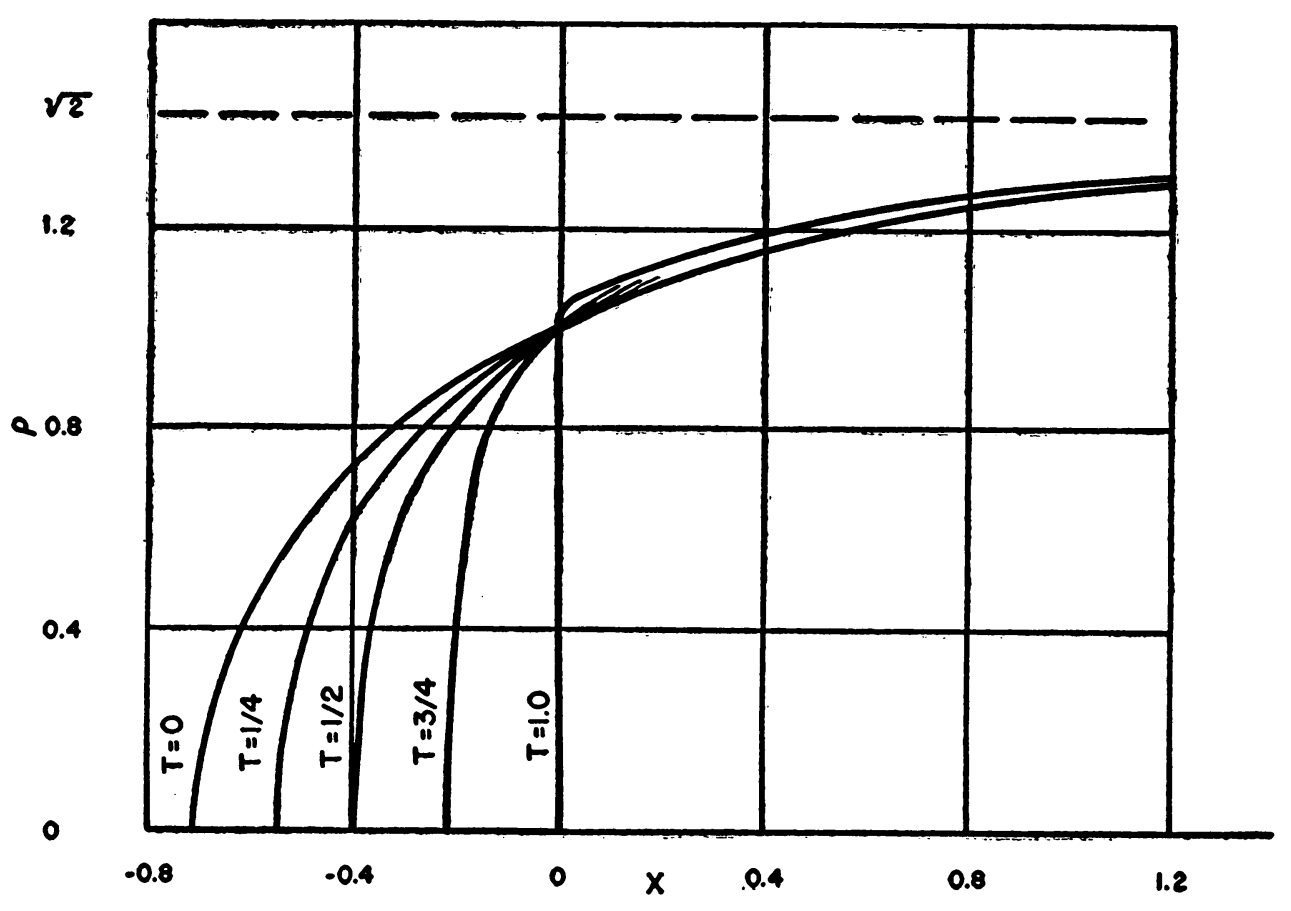

FIg. 2.

In the notation of Legendre,

$$
E\left(M, k^{\prime}\right)=E\left(k^{\prime}, \varphi\right)
$$

where $M$ and $\varphi$ are connected by (2.11).

Using the addition theorem in each case, we see that en $\delta$ is negative imaginary, while $d n \delta$ is positive. Thus,

$$
\text { sn } \delta=l / k, \quad \text { cn } \delta=-i k^{-1}\left(l^{2}-k^{2}\right)^{1 / 2}, \quad \operatorname{dn} \delta=\left(1-l^{2}\right)^{1 / 2}
$$

and

$$
k^{2} \operatorname{sn} \delta \operatorname{cn} \delta \operatorname{dn} \delta=-i l\left(1-l^{2}\right)^{1 / 2}\left(l^{2}-k^{2}\right)^{1 / 2} .
$$


It follows from (2.3) that

$$
I= \pm \int_{0}^{\infty} e^{-|\xi| t} J_{1}(t) J_{1}(\eta t) t^{-1} d t+ \begin{cases}0, & \xi \leq 0 \\ \frac{1}{\eta}, & \xi \geq 0\end{cases}
$$

is continuous at $\xi=0, \eta>1$ having the value $(2 \eta)^{-1}$. Then for $\eta>1$, substituting back into (2.5) and (2.3) and replacing $K-E$ wherever it occurs by $k^{2} D$, we obtain

$$
I=\frac{1}{2 \eta}+\frac{k \xi}{\pi \eta^{1 / 2}}\left[D+\frac{(\eta-1)^{2}}{4 \eta} K\right]+\frac{\eta^{2}-1}{2 \pi \eta}\left[k^{2} D F\left(k^{\prime}, \varphi\right)-K E\left(k^{\prime}, \varphi\right)\right] .
$$

Since both sides vary continuously through $\eta=1$, (2.18) must hold also for $\eta<1$, $\xi \neq 0$. Finally, substituting into (1.8),

$T(\xi, \eta)=\frac{1}{\eta^{2}}+\frac{2 k \xi}{\pi \eta^{3 / 2}}\left[D+\frac{(\eta-1)^{2}}{4 \eta} K\right]+\frac{\eta^{2}-1}{\pi \eta^{2}}\left[k^{2} D F\left(k^{\prime}, \varphi\right)-K E\left(k^{\prime}, \varphi\right)\right]$,

where $\varphi=\arctan \left[\xi(\eta-1)^{-1}\right]$, with $\varphi=0$ at $\xi=0, \eta>1$. In the region outside the disc, $\varphi$ runs from $-\pi$ to $\pi$. For purposes of calculation, we have the following less compact form of $T(\xi, \eta)$ :

$$
\begin{aligned}
& \eta \geq 1, \\
& T(\xi, \eta)=\frac{1}{\eta^{2}}+\frac{2 k \xi}{\pi \eta^{3 / 2}}\left[D+\frac{(\eta-1)^{2}}{4 \eta} K\right] \\
& \mp \frac{\eta^{2}-1}{\pi \eta^{2}}\left[k^{2} D F\left(k^{\prime}, \bar{\varphi}\right)-K E\left(k^{\prime}, \bar{\varphi}\right)\right]\left\{\begin{array}{l}
\xi \leq 0 \\
\xi \geq 0
\end{array}\right. \\
& \eta \leq 1, \\
& T(\xi, \eta)= \pm 1+\frac{2 k \xi}{\pi \eta^{3 / 2}}\left[D+\frac{(\eta-1)^{2}}{4 \eta} K\right] \\
& \mp \frac{1-\eta^{2}}{\pi \eta^{2}}\left[k^{2} D F\left(k^{\prime}, \bar{\varphi}\right)-K E\left(k^{\prime}, \bar{\varphi}\right)\right]+ \begin{cases}0, & \xi \leq 0 \\
2 \eta^{-2}, & \xi \geq 0\end{cases}
\end{aligned}
$$

where $\bar{\varphi}=\arctan \left|\xi(\eta-1)^{-1}\right|, 0 \leq \bar{\varphi} \leq \pi / 2$. We obtain (2.20) from (2.19) using the relations $F\left(k^{\prime}, 2 \pi+\varphi\right)=2 K^{\prime}+F\left(k^{\prime}, \varphi\right), E\left(k^{\prime}, 2 \pi+\varphi\right)=2 E^{\prime}+E\left(k^{\prime}, \varphi\right)$, and the Legendre relation $K E^{\prime}+E K^{\prime}-K K^{\prime}=\pi / 2$.

At $T=0,0 \leq \rho \leq 2^{1 / 2}$,

$$
\left(\frac{\partial T}{\partial x}\right)_{\rho}=-\frac{8}{3}\left(\rho^{2}-1\right)^{-1}\left(2-\rho^{2}\right)^{-1 / 2} .
$$

This result is most easily obtained by going back to (1.8) and (1.12) and making the substitution $\xi=x T^{-1 / 2}, \eta=\rho T^{-1 / 2}$. 


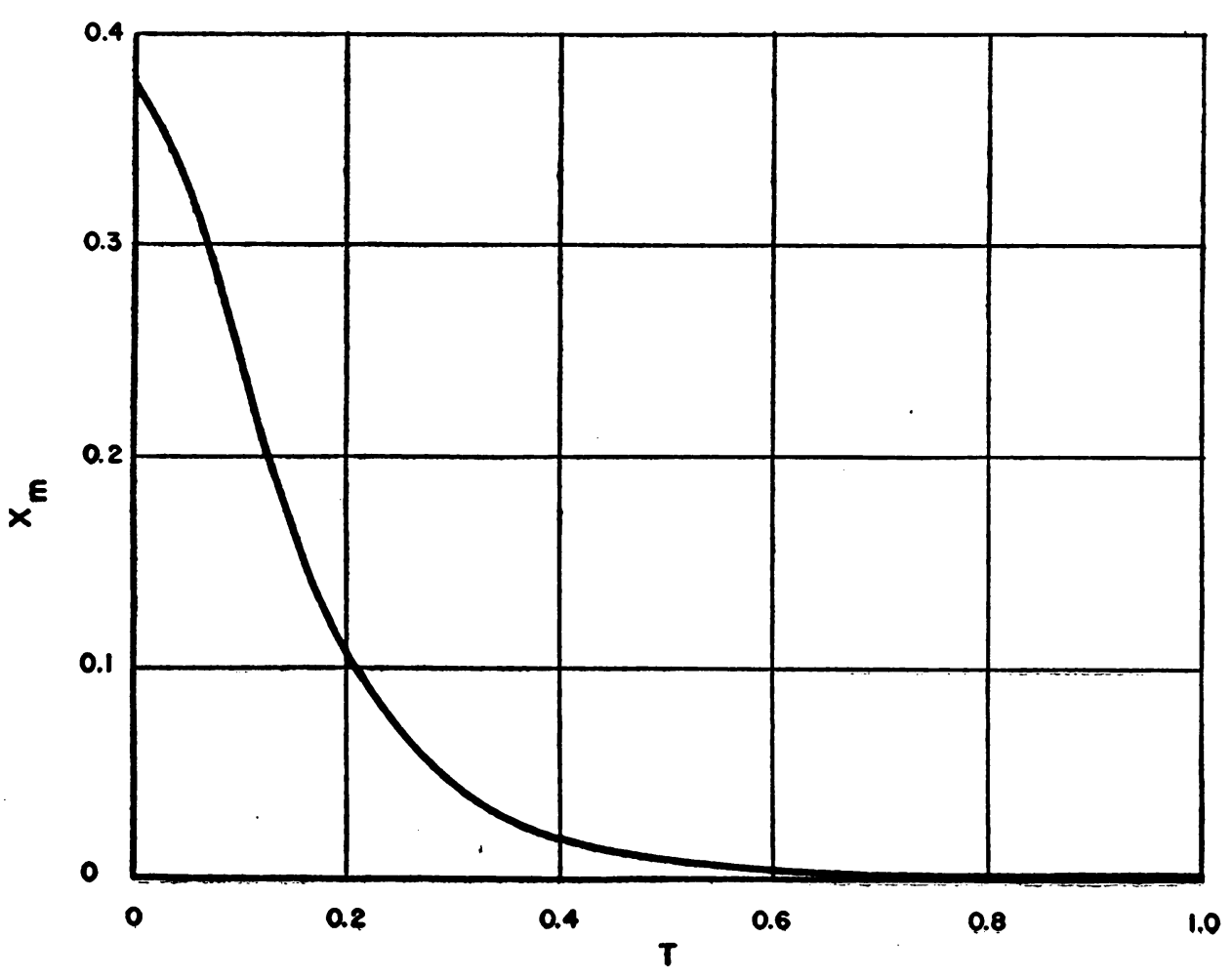

FIG. 3.

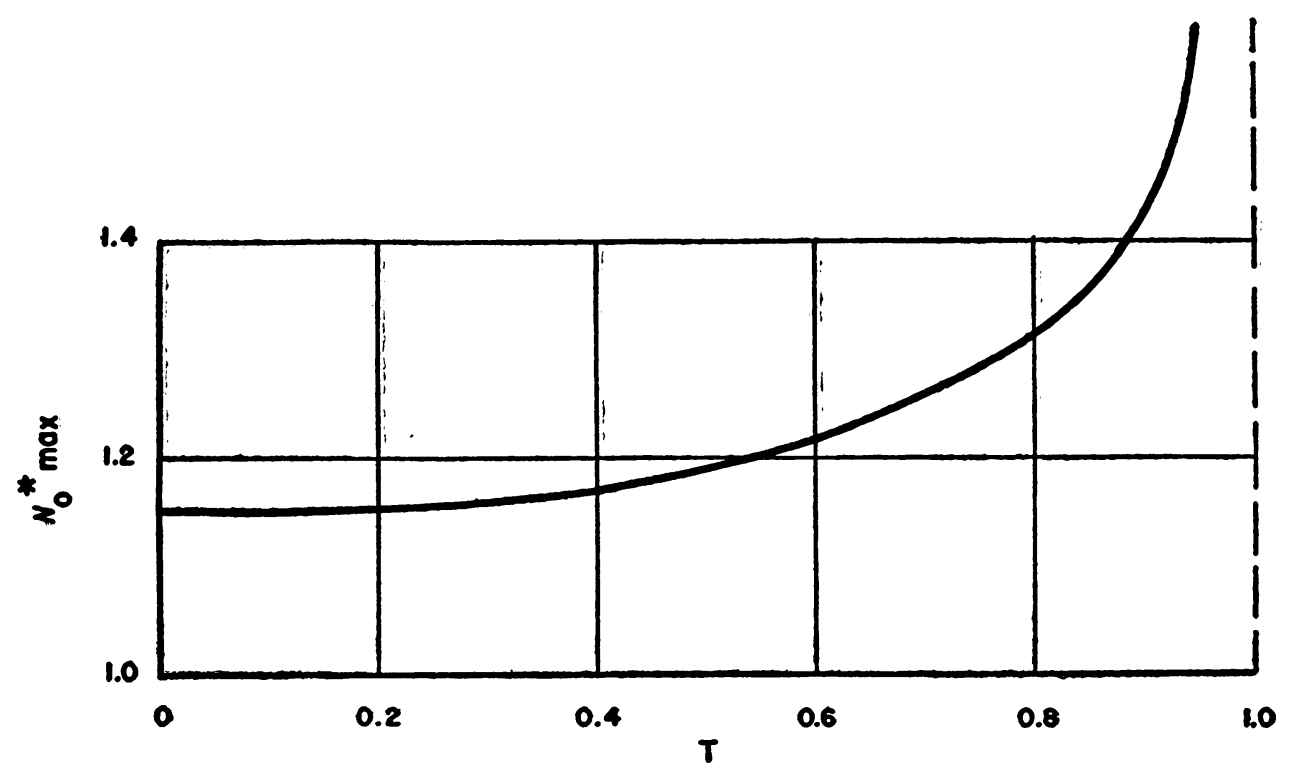

FIG. 4. 
As seen in Fig. 1, the point $T=1, x=0$ is the intersection of all curves $\rho=$ constant for $0 \leq \rho \leq 1$. At this point,

$$
\left(\frac{\partial T}{\partial x}\right)_{\rho}=16 \pi^{-1} C(1+\rho)^{-3}
$$

where $k^{2}=4 \rho(1+\rho)^{-2}$.

3. Calculation of the velocity components. By partial integration, noting that $d J_{1}(Z) / d z=J_{0}(z)-J_{1}(z) z^{-1}$, we have

$$
\begin{array}{r}
\eta \int_{0}^{\infty} e^{-\xi t} J_{1}(t)\left[J_{0}(\eta t)-J_{1}(\eta t)(\eta t)^{-1}\right] d t=\int_{0}^{\infty} e^{-\xi t} J_{1}(t) \frac{\partial}{\partial t} J_{1}(\eta t) d t \\
=-\int_{0}^{\infty} e^{-\xi t} J_{1}(\eta t)\left[-\xi J_{1}(t)+J_{0}(t)-J_{1}(t) t^{-1}\right] d t, \quad \xi \geq 0 .
\end{array}
$$

Further,

$$
\begin{aligned}
\eta \int_{0}^{\infty} e^{-\xi t} J_{0}(t) J_{1}(\eta t) d t & =-\int_{0}^{\infty} e^{-\xi t} J_{0}(t) \frac{\partial}{\partial t} J_{0}(\eta t) d t \\
& =1+\int_{0}^{\infty} e^{-\xi t} J_{0}(\eta t)\left[-\xi J_{0}(t)-J_{1}(t)\right] d t
\end{aligned}
$$

Thus, we establish the identity

$$
\begin{gathered}
\left(1-\eta^{2}\right) \int_{0}^{\infty} e^{-\xi t} J_{0}(\eta t) J_{1}(t) d t=1-2 \eta \int_{0}^{\infty} e^{-\xi t} J_{1}(\eta t) J_{1}(t) t^{-1} d t \\
-\xi \eta \int_{0}^{\infty} e^{-\xi t} J_{1}(\eta t) J_{1}(t) d t-\xi \int_{0}^{\infty} e^{-\xi t} J_{0}(\eta t) J_{0}(t) d t .
\end{gathered}
$$

By Watson [7, p. 389],

$$
\int_{0}^{\infty} e^{-\xi t} J_{n}(\eta t) J_{n}(t) d t=\pi^{-1} \eta^{-1 / 2} Q_{n-1 / 2}\left(\frac{1+\xi^{2}+\eta^{2}}{2 \eta}\right)
$$

where $Q_{\nu}(z)$ is the Legendre function of the second kind of order $\nu$. For $\nu+1>0$, we have the integral representation (cf. $[8$, p. 317])

$$
Q_{\nu}(z)=2^{-\nu-1} \int_{-1}^{1}\left(1-t^{2}\right)^{\nu}(z-t)^{-\nu-1} d t ;
$$

substituting $t=2 u^{2}-1$, with $2(1+z)^{-1}=k^{2}$, into this,

$$
\begin{gathered}
Q_{-1 / 2}(z)=k \int_{0}^{1}\left(1-u^{2}\right)^{-1 / 2}\left(1-k^{2} u^{2}\right)^{-1 / 2} d u=k K, \\
Q_{1 / 2}(z)=k^{3} \int_{0}^{1} u^{2}\left(1-u^{2}\right)^{1 / 2}\left(1-k^{2} u^{2}\right)^{-3 / 2} d u .
\end{gathered}
$$

When $z=\left(1+\xi^{2}+\eta^{2}\right)(2 \eta)^{-1}$, we have $2(1+z)^{-1}=4 \eta\left[\xi^{2}+(1+\eta)^{2}\right]^{-1}$, the quantity already defined as $k^{2}$. Substituting $u=\mathrm{sn}(v, k)$ into (3.3), we find that

$$
Q_{1 / 2}(z)=k^{3} \int_{0}^{K} \frac{\operatorname{sn}^{2} v \mathrm{cn}^{2} v}{\operatorname{dn}^{2} v} d v .
$$


We may easily verify the relation

$$
k^{2} \int \frac{\operatorname{sn}^{2} u \mathrm{cn}^{2} u}{\operatorname{dn}^{2} u} d u=-u+\frac{\operatorname{sn} u \operatorname{cn} u}{\operatorname{dn} u}+2 \int \operatorname{sn}^{2} u d u,
$$

from which it follows that

$$
Q_{1 / 2}(z)=k^{3} C
$$

By (1.8),

$$
2 \eta \int_{0}^{\infty} e^{-|\xi| t} J_{1}(\eta t) J_{1}(t) t^{-1} d t= \pm \eta^{2} T(\xi, \eta)+ \begin{cases}0, & \xi \leq 0 \\ 2, & \xi \geq 0 .\end{cases}
$$

Substituting the preceding results into (3.1), with $\xi \geq 0$ replaced by $|\xi|$, we get

$$
\left(1-\eta^{2}\right) \int_{0}^{\infty} e^{-|\xi| t} J_{0}(\eta t) J_{1}(t) d t= \pm 1 \mp \eta^{2} T(\xi, \eta) \pm \frac{k \xi}{\pi \eta^{1 / 2}}\left(K+k^{2} \eta C\right)\left\{\begin{array}{l}
\xi \leq 0, \\
\xi \geq 0 .
\end{array}\right.
$$

Finally, substituting (3.6) and (3.5) into (1.6) and (1.7), we obtain the following expressions for the velocity components:

$$
\begin{aligned}
\lambda^{-2}\left(u^{*}-1\right) & =\left(1-\eta^{2}\right)^{-1}\left[\eta^{2} T(\xi, \eta)-k \xi\left(\pi \eta^{1 / 2}\right)^{-1}\left(K+k^{2} \eta C\right)-1\right], \\
\lambda^{-2} v^{*} & =k^{3}\left(\pi \eta^{1 / 2}\right)^{-1} C .
\end{aligned}
$$

4. The velocity maximum. For the function $R(\xi, \eta)=2^{-1} u_{0}^{*}\left(\partial w_{0}^{* 2} / \partial \xi\right)_{T}$, we find

$$
R(\xi, \eta)=a u_{0}^{* 2}+b u_{0}^{*} v_{0}^{*}+c v_{0}^{*^{2}},
$$

where

$$
\begin{gathered}
a=\left(2 \pi k \eta^{1 / 2}\right)^{-1}\left(\eta^{2}-1\right)^{-1}\left\{\left[4 \eta+\left(2-2 \eta+\xi^{2}\right) k^{2}\right] K\right. \\
\left.-\left(2 \eta k^{\prime 2}\right)^{-1}\left[8 \eta^{2}+2\left(\xi^{2} \eta-4 \eta^{2}\right) k^{2}+\xi^{2}(1-\eta) k^{4}\right] E\right\}, \\
b=2 \eta\left(1-\eta^{2}\right)^{-2}\left\{T(\xi, \eta)-1-\xi\left(2 \pi k \eta^{5 / 2}\right)^{-1}\left\{\left[4 \eta^{3}-\left(1-4 \eta^{2}+2 \eta^{3}+\eta^{4}\right) k^{2}\right] K\right.\right. \\
\left.\left.-\left(2{k^{\prime}}^{2}\right)^{-1}\left[8 \eta^{3}-2\left(1-2 \eta^{2}+4 \eta^{3}+\eta^{4}\right) k^{2}+\left(1-\eta^{2}\right)^{2} k^{4}\right] E\right\}\right\}, \\
c=-\left(2 \pi k \eta^{3 / 2}\right)^{-1}\left\{\left[4-(2+\eta) k^{2}\right] K-\left(2{k^{\prime}}^{2}\right)^{-1}\left[8-(8+2 \eta) k^{2}+(1+\eta) k^{4}\right] E\right\} .
\end{gathered}
$$

Near $T=0$, the equation of the profile $T=$ constant is

$$
x=\left(\rho^{2}-1\right)\left(2-\rho^{2}\right)^{-1 / 2}-(3 / 8)\left(\rho^{2}-1\right)\left(2-\rho^{2}\right)^{1 / 2} T+O\left(T^{2}\right) .
$$

The coefficient of $T$ is the reciprocal of $(2.21)$, and $x=\left(\rho^{2}-1\right)\left(2-\rho^{2}\right)^{-1 / 2}$ is the equation of the Blasius-Fuhrmann half-body.

Expressing $\psi^{*}$ in a similar form for $T=\lambda^{-2}$ near zero and computing the velocity components, we obtain

$$
w_{0}^{*}=(\rho / 2)\left(8-3 \rho^{2}\right)^{1 / 2}+(3 / 8)\left(2-\rho^{2}\right)^{3}\left(3 \rho^{2}-2\right)\left(8-3 \rho^{2}\right)^{-1} T+O\left(T^{2}\right) .
$$


From (4.5) and (4.6),

$$
\begin{gathered}
x_{m}=6^{-1 / 2}-(13 / 48) 6^{1 / 2} T+O\left(T^{2}\right), \\
w_{0 \max }^{*}=2 / 3^{1 / 2}+O\left(T^{2}\right) .
\end{gathered}
$$

The $x$-intercept $x_{0}(T)$ of the profile is given by

$$
x_{0}(T)=(T-1)\left(2-T^{2}\right)^{-1 / 2} \text {. }
$$

Combining (50) with Fig. 3, we can plot $x_{m}$ measured from the nose of the half-body as a function of $T$.

\section{REFERENCES}

[1] A. Weinstein, On axially symmetric flows, Q. Appl. Math. 5, 429-444 (1948).

[2] E. Beltrami, Opère matematiche, vol. 3, U. Hoepli, Milano, 1911, pp. 349-382.

[3] A. G. Webster, Partial differential equations of mathematical physics, Hafner-Stechert, New York 1933.

[4] H. Lamb, Hydrodynamics, Cambridge University Press, 1932.

[5] H. Weber, J. reine angew. Math., 75, 88.

[6] E. Jahnke and F. Emde, A table of functions, Dover Publications, New York, 1943.

[7] G. N. Watson, Theory of Bessel functions, Macmillan Co., New York, 1944.

[8] E. T. Whittaker and G. N. Watson, Modern analysis, Cambridge University Press, 1927. 